\title{
Measuring Condensate Fraction in Superconductors
}

\author{
Sudip Chakravarty and Hae-Young Kee \\ Department of Physics and Astronomy, University of California Los Angeles \\ Los Angeles, CA 90095-1547
}

(May 19, 2018)

\begin{abstract}
An analysis of off-diagonal long-range order in superconductors shows that the spin-spin correlation function is significantly influenced by the order if the order parameter is anisotropic on a microscopic scale. Thus, magnetic neutron scattering can provide a direct measurement of the condensate fraction of a superconductor. It is also argued that recent measurements in high temperature superconductors come very close to achieving this goal.
\end{abstract}

PACS: 74.72.-h, 75.50.Ee, 74.20.Mn

Off-diagonal long-range order (ODLRO) [1]2] provides an intriguing characterization of superconductors and superfluids for which the order parameter signifies a unique coherence property of a macroscopic quantum system corresponding to the spontaneous breakdown of global gauge symmetry. It is therefore unfortunate that direct measurements of this order parameter are few and far between [3].

For BCS superconductors, all definitions of superconductivity are directly linked to the energy gap in the single particle excitation spectrum. Therefore, the observation of the gap, which is abundant, is virtually the same as the observation of ODLRO. In contrast, the high temperature superconductors sometimes exhibit an apparent gap at temperatures well above the superconducting transition temperature, $T_{c}$, similar to the gap seen below $T_{c}$ [4]. In those instances, the presence of a gap is not the same as the presence of ODLRO, and it becomes necessary to explore ODLRO in more general terms, not restricted by the BCS theory.

The purpose of the present Letter is to offer some insight into the question of ODLRO in high temperature superconductors and to indicate experiments that may shed light on this topic. In fact, we argue that recent magnetic neutron scattering measurements come tantalizingly close to accomplishing this goal [5]. We show that the instantaneous spin-spin correlation function is influenced by the existence of ODLRO. This is partially because the spin operators are composite Fermion operators, and the correlation function can acquire anomalous expectation value, but also because the anisotropic order parameter strongly enhances the effect. We also deduce that strong intercation effects are necessary for the magnitude of the effect to be observable.

a. Summary of experiments: We begin with a brief summary of experiments. The magnetic neutron scattering cross section is proportional to the equilibrium dynamic structure factor, $\mathcal{S}(\mathbf{k}, \omega)$. The corresponding instantaneous structure factor, $\mathcal{S}(\mathbf{k})=\int_{-\infty}^{\infty} d \omega \mathcal{S}(\mathbf{k}, \omega)$, is

$$
\mathcal{S}(\mathbf{k})=v_{0} \sum_{\mathbf{l}} e^{i \mathbf{k} \cdot \mathbf{R}_{\mathbf{l}}}\langle\mathbf{S}(0) \cdot \mathbf{S}(\mathbf{l})\rangle
$$

where $v_{0}$ is the volume of the unit cell, and $\mathbf{S}$ is the spin operator.

For a class of bilayer cuprate superconductors, $\mathrm{YBa}_{2} \mathrm{Cu}_{3} \mathrm{O}_{6+\delta}$ and $\mathrm{Bi}_{2} \mathrm{Sr}_{2} \mathrm{CaCu}_{2} \mathrm{O}_{8+\delta}$, corresponding to optimal doping, the $\mathcal{S}(\mathbf{k}, \omega)$ has a resolution limited resonance peak, at an energy $\approx 40 \mathrm{meV}$ and the special wavevector $\mathbf{k}=\mathbf{q}=(\pi / a, \pi / a, \pi / c)$, for $T<T_{c}$; $a$ and $c$ are the lattice constants. More precisely, the $\mathcal{S}(\mathbf{k}, \omega)$ can be classified into parts that are even and odd with respect to the individual layers of a bilayer. The resonance appears in the odd channel $\left(q_{z}=\pi / c\right)$, and its energy is almost independent of temperature, but its intensity vanishes for $T>T_{c}$. For underdoped superconductors, there is also a peak for $T>T_{c}$, but its character is quite different, as we comment below.

The existence of the resonance peak below $T_{c}$ allows us to isolate a distinct part of the instantaneous structure factor by integrating with respect to frequency across the resonance, which has a simple theoretical interpretation in terms of the condensate fraction, as we shall see. In this sense, the resonance peak plays an essential role; in its absence, it would be difficult to obtain the same information, as in $\mathrm{La}_{2-\delta} \mathrm{Sr}_{\delta} \mathrm{CuO}_{4}$.

b. The Off-diagonal long-range order: The ODLRO for a singlet superconductor is defined as a property of the two particle density matrix, which is

$\rho^{(2)}(1 \uparrow, 2 \downarrow ; 3 \uparrow, 4 \downarrow) \equiv\left\langle\psi_{\uparrow}^{\dagger}\left(\mathbf{x}_{1}\right) \psi_{\downarrow}^{\dagger}\left(\mathbf{x}_{2}\right) \psi_{\downarrow}\left(\mathbf{x}_{4}\right) \psi_{\uparrow}\left(\mathbf{x}_{3}\right)\right\rangle$

where $\psi_{\uparrow}^{\dagger}(\mathbf{x})$ is the creation operator of the Fermion field, of spin up and location $\mathbf{x}$. Its existence implies that

$$
\begin{aligned}
\mid \rho^{(2)}(1 \uparrow, 2 \downarrow ; 3 \uparrow, 4 \downarrow) & -\Phi\left(\mathbf{x}_{1} \uparrow, \mathbf{x}_{2} \downarrow\right)^{*} \Phi\left(\mathbf{x}_{3} \uparrow, \mathbf{x}_{4} \downarrow\right) \mid \\
& \leq\left(\frac{N}{V}\right) \gamma\left(\left|\mathbf{x}_{1}-\mathbf{x}_{3}\right|,\left|\mathbf{x}_{2}-\mathbf{x}_{4}\right|\right),
\end{aligned}
$$

where the the non-negative function $\gamma\left(r_{1}, r_{2}\right)$, which is independent of $N$, tends to zero if either $r_{1}$ or $r_{2}$ tends to $\infty$.

Following Ref. [1], one can show that in the thermodynamic limit, the number of particles $N \rightarrow \infty$ and the volume $V \rightarrow \infty$ such that $(N / V) \rightarrow n$, the function $\Phi$ is the eigenfunction of the two particle density matrix corresponding to the largest eigenvalue $\lambda_{M}$ [6], given by 


$$
\lambda_{M}=\int d \mathbf{x}_{1} d \mathbf{x}_{2}\left|\Phi\left(\mathbf{x}_{1} \uparrow, \mathbf{x}_{2} \downarrow\right)\right|^{2},
$$

where $\lambda_{M} / N$ is the condensate fraction defined to be the fraction of electrons participating in pairing, not to be mistaken to be the superfluid density, $n_{s}$, which is the stiffness with respect to an imposed twist of the order parameter. Hence, in the thermodynamic limit, the following spectral decomposition of the density matrix holds for all separations:

$$
\begin{aligned}
\rho^{(2)}(1 \uparrow, 2 \downarrow ; 3 \uparrow, 4 \downarrow) & =\Phi\left(\mathbf{x}_{1} \uparrow, \mathbf{x}_{2} \downarrow\right)^{*} \Phi\left(\mathbf{x}_{3} \uparrow, \mathbf{x}_{4} \downarrow\right) \\
& +G\left(\mathbf{x}_{1} \uparrow, \mathbf{x}_{2} \downarrow ; \mathbf{x}_{3} \uparrow, \mathbf{x}_{4} \downarrow\right) .
\end{aligned}
$$

The function $G$ vanishes when the separation between the groups $\left(\mathbf{x}_{1}, \mathbf{x}_{2}\right)$ and $\left(\mathbf{x}_{3}, \mathbf{x}_{4}\right)$ tends to $\infty$. While the first term of Eq. (5) vanishes above $T_{c}$, the second term continues smoothly to the normal state above $T_{c}$.

For a translationally invariant system corresponding to a condensate of zero total momentum, we can write $\Phi\left(\mathbf{x}_{1} \uparrow, \mathbf{x}_{2} \downarrow\right) \equiv \Phi\left(\mathbf{x}_{1}-\mathbf{x}_{2}\right)(|\uparrow \downarrow\rangle-|\downarrow \uparrow\rangle) / \sqrt{2}$, where $\Phi(\mathbf{x})=\Phi(-\mathbf{x})$.

For a BCS superconductor (in an abbreviated notation),

$$
\rho^{(2)}=\Phi_{0}\left(\mathbf{x}_{12}\right)^{*} \Phi_{0}\left(\mathbf{x}_{34}\right)+g_{0}\left(\mathbf{x}_{13}\right)^{*} g_{0}\left(\mathbf{x}_{24}\right),
$$

where the BCS function $\Phi_{0}\left(\mathbf{x}_{12}\right) \equiv \Phi_{0}\left(\mathbf{x}_{1}-\mathbf{x}_{2}\right)$ is a function of the separation between the mates of a Cooper pair. Its integral $(1 / N) \int d \mathbf{x}\left|\Phi_{0}(\mathbf{x})\right|^{2}$ is the condensate fraction. In BCS theory, and in the absence of disorder, $n_{s}=n$, while the condensate fraction is $\approx N(0) \Delta$, where $\Delta$ is the gap, and $N(0)$ is the density of states at the Fermi energy. As $T \rightarrow T_{c}$, the condensate fraction tends to zero as $\Delta^{2} N(0) / T_{c}$, similar to $n_{s}$. In general, these are distinct concepts, however.

The BCS function $g_{0}(\mathbf{x})$ is the parallel spin correlation function modified by the presence of a gap, which smoothly continues to the normal state. In contrast, the function $\Phi_{0}(\mathbf{x})$ vanishes in the normal state.

The discussion above can be summarized as follows: (1) the spectral decomposition in Eq. (5) is general and does not depend on the BCS theory; (2) the order parameter function $\Phi$ includes, in principle, all effects of electron-electron interaction; (3) in common with BCS theory, $\lambda_{M}$ vanishes above $T_{c}$, while the contribution due to $G$ continues smoothly above $T_{c}$; (4) as in BCS theory, the function $G$ must be a global gauge singlet.

c. The spin-spin correlation function: From here on we shall abandon the continuum notation and adopt the more natural lattice notation. Assuming that the spin rotational symmetry is unbroken, we note that

$$
\left\langle\mathbf{S}\left(\mathbf{l}_{1}\right) \cdot \mathbf{S}\left(\mathbf{l}_{2}\right)\right\rangle=\frac{3}{4} \delta_{1,2} n_{o}-\frac{3}{2} \rho^{(2)}(1 \uparrow, 2 \downarrow ; 2 \uparrow, 1 \downarrow),
$$

where $n_{o}$ is the average occupation of a site. Making use of the spectral decomposition of the two-particle density matrix, we can write

$$
\begin{aligned}
\left\langle\mathbf{S}\left(\mathbf{l}_{1}\right) \cdot \mathbf{S}\left(\mathbf{l}_{2}\right)\right\rangle & =\frac{3}{4} \delta_{\mathbf{l}_{1}, \mathbf{l}_{2}} n_{o}-\frac{3}{2}\left[\Phi\left(\mathbf{l}_{1} \uparrow, \mathbf{l}_{2} \downarrow\right)^{*} \Phi\left(\mathbf{l}_{2} \uparrow, \mathbf{l}_{1} \downarrow\right)\right. \\
& \left.+G\left(\mathbf{l}_{1} \uparrow, \mathbf{l}_{2} \downarrow ; \mathbf{l}_{2} \uparrow, \mathbf{l}_{1} \downarrow\right)\right] .
\end{aligned}
$$

Then remembering spin rotational symmetry and using Eqs. 8 and 1, we get

$$
\mathcal{S}(\mathbf{k})=\frac{3}{4} v_{0} n_{0}-\frac{3}{2} v_{0} \sum_{\mathbf{l}} e^{i \mathbf{k} \cdot \mathbf{R}_{\mathbf{1}}}\left[\lambda_{M}|f(\mathbf{l})|^{2}+G(\mathbf{l})\right],
$$

where $f(\mathbf{l})$ is now assumed to be normalized $\left(v_{0} \sum_{\mathbf{l}}|f(\mathbf{l})|^{2}=1\right)$. From the perspective of ODLRO, the summation variable $\mathbf{l}$ in Eq. (9) is a degree of freedom internal to the Cooper pair, denoting the separation between its mates. In the absence of simultaneous diagonal long range order, $G(\mathbf{l})$ is a short ranged quantity, which is qualitatively unaffected by superconductivity, essentially because the short-distance electron-electron interaction is similar in both the normal and the superconducting phases. The underdoped cuprates, discussed below, are more complex.

We first consider a single $\mathrm{CuO}$-plane, ignoring the fluctuations that destroy ODLRO at any finite temperature, $T$; the coupling between the planes is discussed below in section $(f)$. The wavevectors $\mathbf{k}$ and $\mathbf{q}$ are to be interpreted as two-dimensional $(2 D)$ vectors, strictly $\mathbf{k}_{\|}$and $\mathbf{q}_{\|}$, until the section $(f)$; similarly, $\mathbf{R}_{\mathbf{l}}$ is to be interpreted as a $2 D$ vector. We hope that this is not a cause for confusion.

d. Optimally doped superconductors: Consider first the case of optimally doped superconductors for which $\mathcal{S}(\mathbf{k})$ is peaked at $\mathbf{k}=\mathbf{q}$ for $T<T_{c}$, but not so for $T>T_{c}$. Here, $\mathbf{q}$ is the vector $(\pi / a, \pi / a)$. In Eq. (9), the exponential factor changes sign rapidly on the scale of a lattice spacing when $\mathbf{k}=\mathbf{q}$. For any functions $|f(\mathbf{l})|^{2}$ and $G(\mathbf{l})$ that are smooth on the scale of a lattice spacing, the sum will be negligible.

An order parameter function $f(\mathbf{l})$ such that $|f(\mathbf{l})|^{2}$ vanishes when the vector $\mathbf{R}_{\mathbf{l}}$ connects sites belonging to the same sublattice of a bipartite square lattice [7] can lead to a peak in $\mathcal{S}(\mathbf{k})$, because

$$
\sum_{\mathbf{l}} e^{i \mathbf{q} \cdot \mathbf{R}_{\mathbf{l}}}|f(\mathbf{l})|^{2}=-\sum_{\mathbf{l} \in A}|f(\mathbf{l})|^{\mathbf{2}},
$$

where the sum on the right hand side is over the sublattice $A$. Clearly, a $d$-wave order parameter satisfies this criterion, but so does an anisotrpic $s$-wave order. The distinction between them can only be made on energetic grounds. The range of $f(\mathbf{l})$ is the "size of the Cooper pair".

From the argument given above, we expect $G(\mathbf{l})$ to be largely unaffected by superconductivity. We can then focus on the quantity

$$
\Delta \mathcal{S}(\mathbf{k}+\mathbf{q})=\frac{3}{2} v_{0} \lambda_{M} \sum_{\mathbf{l} \in A} e^{i \mathbf{k} \cdot \mathbf{R}_{1}}|f(\mathbf{l})|^{2} .
$$


Then, noting that $f(\mathbf{l})$ is normalized, we get

$$
\frac{\Delta \mathcal{S}(\mathbf{q})}{N}=\frac{3}{2} \frac{\lambda_{M}}{N}
$$

The inverse $\mathbf{k}$-width of $\Delta \mathcal{S}(\mathbf{k}+\mathbf{q})$ is the size of the Cooper pair. This result is consistent with a general inequality derived in Ref. [8]. The authors of Ref. [8] have further approximated the inequality to obtain $\Delta \mathcal{S}(\mathbf{q}) \geq$ $(3 / 2)|\Delta|^{2} / x$, where $\Delta$ is the nearest-neighbor $d$-wave order parameter, and $x$ is the concentration of holes.

If $\lambda_{M}$ were of the magnitude predicted for a BCS superconductor, that is, $N(0) \Delta$, the intensity would be below the sensitivity limit of current neutron scattering measurements. That the intensity is readily detectable means that the condensate fraction in the high temperature superconductors is more than an order of magnitude greater than that predicted in BCS theory. For tightly bound noninteracting Cooper pairs, acting as molecules, the condensate density would be the full density $n$.

e. Underdoped superconductors: For underdoped superconductors [9], $\mathcal{S}(\mathbf{k})$ is peaked above $T_{c}$ at the wavevector $\mathbf{q}$, but the growth of the intensity is gradual, increasing by about $20 \%$ from $300 \mathrm{~K}$ to $T_{c} \sim 50 \mathrm{~K}$, seen in both even and odd channels. In contrast, the intensity in the odd channel rises by almost a factor of 2 below $T_{c}$. It is very natural therefore to conclude that $G(\mathbf{l})$ is of very different character than $f(\mathbf{l})$. Thus, even in the underdoped case, it is possible to separate out the effect of $G(\mathbf{l})$ and to focus on the contribution solely due to $f(\mathbf{l})$. Before interpreting the data in terms of condensate fraction, we remark briefly on the peaked nature of $\mathcal{S}(\mathbf{k})$ above $T_{c}$; there are a number of distinct possibilities.

The first possibility is that the spin correlations are described by a quantum disordered state for which the structure factor is peaked at the commensurate wavevector q. For such a quantum disordered state, it is difficult, however, to construct a $d$-wave modulation of the gap in the single particle excitation spectrum as seen in photoemission experiments 10$]$.

The second possibility is superconducting fluctuations above $T_{c}$. While such fluctuations must exist above $T_{c}$, especially in the underdoped regime, it is unlikely that they produce a peaked $\mathcal{S}(\mathbf{k})$ at temperatures as high as $300 \mathrm{~K}$.

The third possibility is a flux state for which the saddle point result [11] for the insulator is remarkably isomorphic to the BCS result for a superconductor, and $\mathcal{S}(\mathbf{k})$ is peaked at the wavevector $\mathbf{q}$, for essentially the same reason. Unfortunately, it is difficult to see why this should hold as the system is doped with holes, although it has been argued [12] that the notions of the flux state should continue to hold for the doped case.

The interpretation of $\Delta \mathcal{S}(\mathbf{q})$, in terms of the condensate fraction has interesting consequences for the quantum phase transition as a function of the hole concentration, $x$, hence for the global phase diagram. As $x \rightarrow x_{c}^{+}$, typically $\sim 5 \%$, the superfluid density is known to vanish 13], but the condensate fraction increases as shown in Fig. 1. This is unusual and calls for an explanation.

A possible resolution is that the experimental system cannot be considered pure, at least for $x$ close to $x_{c}$, and that this transition is radically altered by disorder, allowing the condensate fraction to remain finite at the transition. The discontinuous drop in the condensate fraction at $x_{c}$ may suggest a first order transition, but it need not be. There is at least one example in statistical mechanics where it is known that the order parameter vanishes discontinuously, yet the correlation length diverges at the transition 114. The role of disorder at this quantum phase transition may then be of crucial importance, and any discussion of a quantum critical point [15] must be reconsidered in this light.

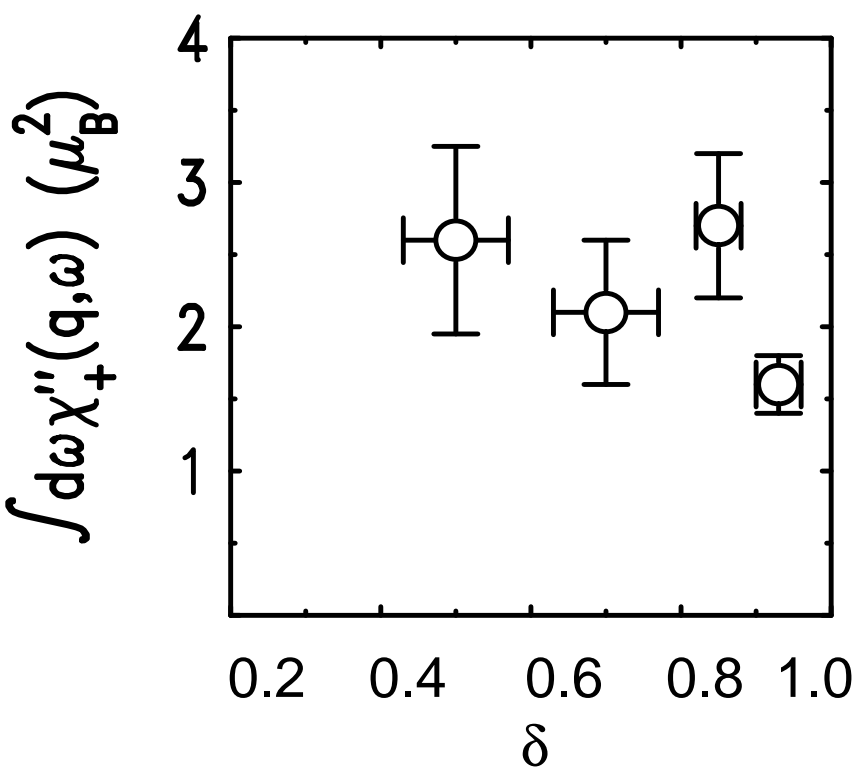

FIG. 1. The imaginary part of the dynamical susceptibility, $\chi_{+}^{\prime \prime}(q, \omega)$ of $\mathrm{YBa}_{2} \mathrm{Cu}_{3} \mathrm{O}_{6+\delta}$ at $10 \mathrm{~K}$ [9]. This is proportional to $\Delta \mathcal{S}(\mathbf{q})$, because, at such low temperatures, the difference between the two is negligible. The subscript + refers to the enhancement over the normal state close to $T_{c}$; the data is for the odd channel, as defined in the text. The parameter $\delta \approx 0.42$ corresponds to the crtical hole concentration $x_{c}$ at which the superconductivity disappears at $T=0$.

There may be another possible resolution [16]. The pseudogap phase may be a discrete broken symmetry phase in disguise, known variously as "flux" or "dimer" ordered state, which has a peaked $\mathcal{S}(\mathbf{k})$. Superconductivity then develops on top of this. Such a broken symmetry state is easily corrupted by disorder, notoriously plentiful in this class of materials, which is why it has been identified experimentally only as a "crossover" in some materials and not at all in others. This crossover will sharpen to an actual phase transition as the material qualities improve. The neutron intensity within the resonance peak is then a superposition of two effects, one 
due to the condensate fraction and the other the due to the discrete broken symmetry.

We cannot, of course, rule out the possibility that future measurements may reveal that the condensate fraction turns around and vanishes continuously at $x_{c}$, especially because it is so difficult to obtain the absolute magnitude of the intensity in the neutron measurements. If this is the case, the interpretation of the quantum critical point at $x_{c}$ will be straightforward. There is some evidence that the intensity may vanish at $x_{c}$. The data shown in Fig. 1 was obtained by integrating over only the positive part of the imaginary part of the susceptibility enhanced over the normal state. Thus, a more complete integration over the frequency will be necessary to obtain the true theoretical value of the condensate fraction.

f. Three-dimensional (3D) and bilayer couplings In bilayer superconductors, the adjacent layers are grouped into CuO-bilayers, which are then very weakly coupled. The small coupling between the bilayers should have a small effect on the $2 D$ spin fluctuations seen above $T_{c}$, and indeed this is consistent with experiments. Likewise, such small couplings should have small effects also on the ordered state at $T=0$. Close to $T_{c}$, small $3 D$ couplings should affect the critical behavior, however, and, below $T_{c}$, these couplings are necessary to establish an order parameter. As far as these weak $3 D$ couplings of groups of bilayers are concerned, qualitatively, little needs to be added to the previous discussion. These comments would be hardly modified if we were considering superconductors in which single layers (instead of groups of bilayers) were coupled by weak $3 D$ coupling.

In contrast, there is evidence that the individual layers within a bilayer are reasonably strongly coupled, thereby splitting the bilayer spin responses that are odd and even with respect to the individual layers. Although the dynamics is beyond the scope of the present paper, we can make a few brief qualitative remarks. For magnetic neutron measurements, the relevant coupling must be an antiferromagnetic superexchange, which must gap the even response function at low frequencies, as in experiments. This can be seen by noting that the spin correlations are approximately described by a bilayer $\sigma$-model [17] and expanding the Euclidean Lagrangian to quadratic order [18]. The magnitude of the bilayer gap is a function of the $\sigma$-model parameters and must depend on doping, as in experiments [9]. As a result, from the $3 D$ dynamic structure factor, $S^{3 \mathrm{D}}(\mathbf{k}, \omega)=\sin ^{2}\left(\frac{k_{z} c}{2}\right) S_{\mathrm{a}}^{2 \mathrm{D}}\left(\mathbf{k}_{\|}, \omega\right)+\cos ^{2}\left(\frac{k_{z} c}{2}\right) S_{\mathrm{s}}^{2 \mathrm{D}}\left(\mathbf{k}_{\|}, \omega\right)$, the symmetric (even) part can be dropped at low frequencies. The integration across the resonance, which appears sharply in the odd (antisymmetric) channel, then gives the required structure factor.

In conclusion, we have investigated the static structure factor for which we could make precise statements, although the experiments on this are far from complete. We hope that future experiments will shed further light on the questions regarding the condensate fraction raised here. It remains to be seen if similar precise statements could be made for the dynamical structure factor as well.

We thank E. Abrahams, R. J. Birgeneau, P. Bourges, R. B. Laughlin, B. Keimer, C. Nayak, and S. -C. Zhang for comments. S. C. acknowledges the grant NSF-DMR9971138. The work of H. -Y. K was conducted under the auspices of the Department of Energy, supported (in part) by funds provided by the University of California for the conduct of discretionary research by Los Alamos National Laboratory. A part of this work was carried out at the Aspen Center for Physics.

[1] O. Penrose and L. Onsagar, Phys. Rev. 104, 576 (1956).

[2] C. N. Yang, Rev. Mod. Phys. 34, 694 (1962).

[3] For a recent review on condensate fraction in ${ }^{4} \mathrm{He}$, see $\mathrm{P}$. Sokol in Bose-Einstein Condensation, edited by A. Griffin, D. W. Snoke, and S. Stringari (Cambridge University Press, Cambridge, 1995).

[4] For a brief review, see, for example, M. Randeria, Varenna Lectures, 1997 cond-mat/9711232.

[5] H. F. Fong et al., Phys. Rev. Lett. 75, 316 (1995); P. Bourges et al., Phys. Rev. B 53, 876 (1996); H. F. Fong et al., Phys. Rev. B 54, 6708 (1996); P. Dai et al., Phys. Rev. Lett. 77, 5425 (1996); H. F. Fong et al., Phys. Rev. Lett. 78, 713 (1997); P. Bourges et al., Europhys. Lett. 38, 313 (1997); H. A. Mook et al., Nature 395, 580 (1998); H. F. Fong et al., Nature 398, 588 (1999).

[6] The eigenvalue $\lambda_{M}$ is assumed to be nondegenerate because these superconductors are not in a superposition of macroscopically distinct ODLRO states.

[7] C. L. Henley, Phys. Rev. Lett. 80, 3590 (1998).

[8] E. Demler, H. Kohno, and S. -C. Zhang, Phys. Rev. B 58, 5719 (1998).

[9] H. F. Fong et al., preprint, cond-mat/9910041; see also, B. Keimer et al., Physica C 282-287, 232 (1997).

[10] F. Ronning et al., Science 282, 2067 (1998).

[11] J. B. Marston and I. Affleck, Phys. Rev. B 39, 11538 (1989).

[12] R. B. Laughlin, Phys. Rev. Lett. 79, 1726 (1997).

[13] See, for example, B. Batlogg in High Temperature Superconductivity, edited by K. Bedell et al., 37 (AddisonWesley, Redwood City, 1990).

[14] See, for example, D. J. Thouless, Phys. Rev. 187, 732 (1969).

[15] R. B. Laughlin, Adv. in Phys. 47, 943 (1998).

[16] S. Chakravarty and R. B. Laughlin, unpublished.

[17] L. Yin, M. Troyer, and S. Chakravarty, Europhys. Lett. 42, 559 (1998).

[18] The even-odd response functions are interchanged in the $\sigma$-model because the unit vector field in this model is related to the spin operator $(-1)^{i+p} \mathbf{S}_{i}^{(p)}$, where $i$ are the sites of the $2 D$ lattice and $p=1,2$ are indices of the layers within a bilayer 17. 